\title{
Whole-genome sequencing of six dog breeds from continuous altitudes reveals adaptation to high-altitude hypoxia
}

\author{
Xiao Gou, ${ }^{1,2,10}$ Zhen Wang, ${ }^{3,4,10}$ Ning Li, ${ }^{2,10}$ Feng Qiu, ${ }^{4,5,10}$ Ze Xu, ${ }^{5}$ Dawei Yan, ${ }^{1}$ \\ Shuli Yang, ${ }^{1}$ Jia Jia, ${ }^{4}$ Xiaoyan Kong, ${ }^{1}$ Zehui Wei, ${ }^{6}$ Shaoxiong Lu, ${ }^{1}$ Linsheng Lian, ${ }^{1}$ \\ Changxin Wu, ${ }^{2}$ Xueyan Wang, ${ }^{1}$ Guozhi Li, ${ }^{1}$ Teng Ma, ${ }^{1}$ Qiang Jiang, ${ }^{1}$ Xue Zhao, ${ }^{1}$ \\ jiaqiang Yang, ${ }^{1}$ Baohong Liu, ${ }^{5}$ Dongkai Wei, ${ }^{5}$ Hong Li, ${ }^{3,4}$ Jianfa Yang, ${ }^{1}$ Yulin Yan, ${ }^{1}$ \\ Guiying Zhao, ${ }^{1}$ Xinxing Dong, ${ }^{1}$ Mingli Li, ${ }^{1}$ Weidong Deng, ${ }^{1}$ Jing Leng, ${ }^{1}$ \\ Chaochun Wei, ${ }^{4,7}$ Chuan Wang, ${ }^{8}$ Huaming Mao, ${ }^{1}$ Hao Zhang, ${ }^{2}$ Guohui Ding, ${ }^{3,4}$ \\ and Yixue $\mathrm{Li}^{3,4,7,9}$
}

\begin{abstract}
${ }^{1}$ College of Animal Science and Technology, Yunnan Agricultural University, Kunming 650201, China; ${ }^{2}$ College of Animal Science and Technology/State Key Laboratory for Agrobiotechnology, China Agricultural University, Beijing 100094, China; ${ }^{3}$ Key Laboratory of Systems Biology, Shanghai Institute for Biological Sciences, Chinese Academy of Sciences, Shanghai 200031, China; ${ }^{4}$ Shanghai Center for Bioinformation Technology, Shanghai Industrial Technology Institute, Shanghai 201203, China; ${ }^{5}$ EG Information Technology Enterprise (EGI), Encode Genomics Biotechnology Co., Ltd., Shanghai 200235, China; ${ }^{6}$ College of Animal Science and Technology, Northwest Agricultural and Forestry University, Yangling 712100, China; ${ }^{7}$ School of Life Science and Technology, Shanghai Jiaotong University, Shanghai 200240, China; ${ }^{8}$ National Center for Protein Science Shanghai, National Facility for Protein Science in Shanghai, Institute of Biochemistry and Cell Biology, Chinese Academy of Sciences, Shanghai 200031, China; ${ }^{9}$ School of Life Science and Technology, Shanghai Tongji University, Shanghai 200092, China
\end{abstract}

\begin{abstract}
The hypoxic environment imposes severe selective pressure on species living at high altitude. To understand the genetic bases of adaptation to high altitude in dogs, we performed whole-genome sequencing of 60 dogs including five breeds living at continuous altitudes along the Tibetan Plateau from 800 to $5100 \mathrm{~m}$ as well as one European breed. More than $150 \times$ sequencing coverage for each breed provides us with a comprehensive assessment of the genetic polymorphisms of the dogs, including Tibetan Mastiffs. Comparison of the breeds from different altitudes reveals strong signals of population differentiation at the locus of hypoxia-related genes including endothelial Per-Arnt-Sim (PAS) domain protein 1 (EPASI) and beta hemoglobin cluster. Notably, four novel nonsynonymous mutations specific to high-altitude dogs are identified at EPASI, one of which occurred at a quite conserved site in the PAS domain. The association testing between EPASI genotypes and blood-related phenotypes on additional high-altitude dogs reveals that the homozygous mutation is associated with decreased blood flow resistance, which may help to improve hemorheologic fitness. Interestingly, EPASI was also identified as a selective target in Tibetan highlanders, though no amino acid changes were found. Thus, our results not only indicate parallel evolution of humans and dogs in adaptation to high-altitude hypoxia, but also provide a new opportunity to study the role of EPAS1 in the adaptive processes.
\end{abstract}

[Supplemental material is available for this article.]

The mechanisms of organismal adaptation to high-altitude hypoxia are of great interest during recent years. Highland wild animals have a long life history at high altitude, and the whole genomes of yak (descendants of wild yak) (Qiu et al. 2012), Tibetan antelope (Ge et al. 2013), snow leopard (Cho et al. 2013), and wild boar (Li et al. 2013) have been sequenced. In contrast, the human settlement history on highland is rather short, which dates from $\sim 25,000$ yr ago (Zhao et al. 2009). Whole-genome genotyping and re-sequencing have been performed for three typical highland

\footnotetext{
${ }^{10}$ These authors contributed equally to this work.

Corresponding authors: maohm@vip.sina.com, zhanghao827@163. com, gwding@sibs.ac.cn, yxli@sibs.ac.cn

Article published online before print. Article, supplemental material, and publication date are at http://www.genome.org/cgi/doi/10.1101/gr.171876.113.
}

populations including Tibetans (Beall et al. 2010; Bigham et al. 2010; Simonson et al. 2010; Yi et al. 2010; Peng et al. 2011; Xu et al. 2011), Andeans (Bigham et al. 2009, 2010), and Ethiopians (Alkorta-Aranburu et al. 2012; Scheinfeldt et al. 2012).

The increased oxygen uptake and delivery are physiological hallmarks of high-altitude adaptation. On one hand, the capacity of oxygen uptake is determined by hemoglobin concentration and oxygen affinity. For example, the Andean highlanders display a high level of hemoglobin concentration (Beall et al. 2002; Beall 2007). http://genome.cshlp.org/site/misc/terms.xhtml). After six months, it is available under a Creative Commons License (Attribution-NonCommercial 4.0 International), as described at http://creativecommons.org/licenses/by-nc/4.0/. 
The high oxygen affinity of hemoglobin is found in many highland animals such as yak (Weber et al. 1988), alpaca (Piccinini et al. 1990), deer mice (Storz et al. 2007; Storz et al. 2009), bar-headed goose (Zhang et al. 1996; Liang et al. 2001), and Andean goose (Jessen et al. 1991). On the other hand, the rate of oxygen delivery is determined by blood flow. For example, although Tibetans maintain a nearly normal level of hemoglobin concentration and a low level of oxygen saturation, they display a high level of blood flow, resulting in the increase of oxygen delivery (Beall et al. 2001; Erzurum et al. 2007).

Whole-genome scans revealed that positive selection for human high-altitude adaptation occurred in the hypoxia-inducible factor (HIF) pathway (Bigham et al. 2009; Beall et al. 2010; Bigham et al. 2010; Simonson et al. 2010; Yi et al. 2010; Peng et al. 2011; Xu et al. 2011; Alkorta-Aranburu et al. 2012; Scheinfeldt et al. 2012), which regulates genes associated with blood physiology. In addition, metabolic pathways may also be involved in the adaptive process of yak (Qiu et al. 2012) and Tibetan antelope (Ge et al. 2013).

Although a lot of studies focused on wildlife and human highlanders, no research was performed on domesticated animals that migrated to the plateau with humans, which represent an adaptation pattern on a short evolutionary time scale of thousands of years. For example, the Tibetan Mastiff is a native dog living in the Tibetan Plateau with an altitude of 3000-6000 m. It is also an ancient dog in the world (Li and Zhang 2012). However, the genetic and physiological mechanisms of its adaptation to high-altitude environments remain elusive.

In this study, we sampled five dog breeds including the Tibetan Mastiff from continuous altitudes along the Ancient Tea Horse Road in southwestern China as well as one European breed. We performed whole-genome sequencing for the dogs and identified candidate genes for high-altitude adaptation using selective sweep mapping. We also measured the hematologic and hemorheologic parameters of the dogs and tested the association between the candidate alleles and blood physiology.

\section{Results}

\section{Genetic diversity and population structure}

We sampled 60 dogs belonging to six breeds living at continuous altitudes (Table 1). To avoid potential kinships, each dog was sampled from one individual village, which is dispersed along the Ancient Tea Horse Road and far away from each other. The sex ratio was kept as 1:1 for each breed. We classified the breeds into three altitude levels, i.e., high (>3000 m), middle (2000-3000 m), and low $(<2000 \mathrm{~m})$. The high-altitude breeds include Tibetan Mastiffs (TM) and Diqing indigenous dogs (DQ). The middle-altitude breed was chosen as Lijiang indigenous dogs (LJ). The low-altitude breeds contain Yingjiang indigenous dogs (YJ), Kunming dogs (KM), and German Shepherds (GS). Grouping multiple breeds living at similar altitudes could contribute to reducing the influence of breedspecific genetic background.

We performed whole-genome sequencing for each individual dog. The coverage depth after genome alignment is $\sim 15 \times$ for each dog (Supplemental Fig. 1; Supplemental Tables 1, 2), resulting in $>150 \times$ for each breed for variant calling (Supplemental Tables $3-$ 5 ). The levels of genome-wide genetic diversity, as measured by Watterson's $\theta$ and pairwise nucleotide diversity $\pi$, are relatively higher in the TM and indigenous breeds (DQ, LJ, and YJ) than those in KM and GS (Table 1; Supplemental Fig. 2), consistent with the fact that the former are more ancient breeds than the latter working dogs that have been domesticated recently (Vonholdt et al. 2010; Wang et al. 2013). In agreement with this, the minor allele frequency (MAF) distribution and Tajima's $D$ indicate a larger proportion of low frequency minor alleles in the ancient breeds than in the modern breeds (Supplemental Fig. 3). Additionally, linkage disequilibrium (LD) and haplotype analyses show that the ancient breeds have a faster decay of pairwise correlation coefficient $\left(r^{2}\right)$ and lower integrated haplotype homozygosity (iHH) (Voight et al. 2006) than the modern breeds (Supplemental Fig. 4).

To examine genetic relationships among the dogs, we conducted principal component analysis (PCA) based on whole-genome SNPs. In the PCA plot, the LJ, YJ, KM, and GS breeds could be separated by the first and second eigenvectors, while TM and DQ are mixed (Fig. 1A). However, when more eigenvectors are incorporated, most dogs in TM and DQ could still be separated (Fig. 1A). These results suggest close but different genetic backgrounds between TM and DQ. To resolve their phylogenetic relationships, we constructed a neighbor-joining (NJ) tree based on the pairwise genetic distances (Fig. 1B). The tree also splits TM and DQ into distinct branches except one dog in TM. Considering possible admixture among the breeds, we further performed population structure analysis with frappe (Tang et al. 2005), which estimates individual ancestry and admixture proportions assuming $K$ ancestral populations (Fig. 1C). When $K=2$, we found a division between the ancient (TM, DQ, LJ, and YJ) and modern breeds (KM and GS). When $K=3$, we found a division between the breeds living at high altitude (TM and DQ) and at middle/low altitude (LJ and YJ). When $K=5$, TM and DQ are separated, though a slight extent of ingression from DQ happened in the TM breed.

\section{Signature of selection associated with high altitude}

To detect the signature of natural selection associated with high altitude, we searched the dog genome for regions with a high coefficient of nucleotide differentiation $F_{\mathrm{ST}}$ among the breeds living at high (TM, DQ), middle (LJ), and low altitude (YJ, KM, GS) (Fig. $2 \mathrm{~A})$. The approach of population differentiation has been widely used to detect selective sweeps based on whole-genome SNPs, such as artificial selection during dog domestication (Axelsson et al. 2013) and diversification (Akey et al. 2010; Vonholdt et al. 2010). We scanned the autosomes with a nonoverlapping 100-kb window and calculated the $F_{\mathrm{ST}}$ value for each window. As previous studies (Axelsson et al. 2013), we focused on the regions with extremely high $Z$-transformed $F_{\mathrm{ST}}$ values $\left[Z\left(F_{\mathrm{ST}}\right)>5\right.$ ] in the genome-wide empirical distribution. In total, 28 unique autosomal regions containing 141 candidate genes were identified (Supplemental Table 6), and five genes of them including EPAS1, MSRB3, HBB, $C D K 2$, and GNB1 belong to the gene ontology (GO) categories "response to oxygen levels" and "response to oxidative stress" (Fig. 2A). To date the sweeps during the migration of dogs from low to high altitude, we also calculated the pairwise $F_{\mathrm{ST}}$ and $Z\left(F_{\mathrm{ST}}\right)$ between the three altitude levels (Supplemental Fig. 5; Supplemental Table 7).

The region with the strongest differentiation signal $\left[Z\left(F_{\mathrm{ST}}\right)=\right.$ 10.83] (Fig. 2A) among the three altitude levels incorporates endothelial Per-Arnt-Sim (PAS) domain protein 1 (EPAS1), a gene encoding the HIF 2A (Patel and Simon 2008). The region is also highly differentiated when the high-altitude breeds were compared with the low- and middle-altitude ones $\left[Z\left(F_{\mathrm{ST}}\right)=10.97\right.$ and 8.02 , respectively] (Supplemental Fig. 5), suggesting that it is involved in a selective sweep during the move of dogs from middle to high altitude (Supplemental Table 7). Interestingly, EPAS1 was also 
Table 1. Sample, sequencing, and polymorphism information for the six dog breeds

\begin{tabular}{|c|c|c|c|c|c|c|}
\hline Breed (abbreviation) & $\begin{array}{c}\text { Sample } \\
\text { size }\end{array}$ & Location & Altitude & $\begin{array}{l}\text { Total coverage } \\
\text { of depth }^{\mathbf{a}}(\times)\end{array}$ & $\begin{array}{l}\text { Watterson's } \\
\theta\left(\times 10^{-3}\right)\end{array}$ & $\begin{array}{l}\text { Nucleotide diversity } \\
\pi\left(\times 10^{-3}\right)\end{array}$ \\
\hline Tibetan Mastiff (TM) & 10 & $\begin{array}{l}\text { Cuomei, Tibet, China }(n=4) \\
\text { Yushu, Qinghai, China }(n=4) \\
\text { Diqing, Yunnan, China }(n=2)\end{array}$ & $\begin{array}{l}5100 \mathrm{~m} \\
4200 \mathrm{~m} \\
3300 \mathrm{~m}\end{array}$ & 154.22 & 1.22 & 1.35 \\
\hline Diqing indigenous dog (DQ) & 10 & Diqing, Yunnan, China & $3300 \mathrm{~m}$ & 154.88 & 1.22 & 1.35 \\
\hline Lijiang indigenous dog (니) & 10 & Lijiang, Yunnan, China & $2400 \mathrm{~m}$ & 154.72 & 1.27 & 1.35 \\
\hline Kunming dog (KM) & 10 & Kunming, Yunnan, China & $1800 \mathrm{~m}$ & 159.23 & 0.99 & 1.15 \\
\hline German Shepherd (GS) & 10 & Kunming, Yunnan, China & $1800 \mathrm{~m}$ & 159.62 & 0.61 & 0.75 \\
\hline Yingjiang indigenous dog $(Y J)$ & 10 & Yingjiang, Yunnan, China & $800 \mathrm{~m}$ & 153.46 & 1.28 & 1.38 \\
\hline
\end{tabular}

${ }^{a}$ Only autosomes were considered for depth and polymorphism calculation.

identified as a selective target in Tibetan people (Beall et al. 2010; Yi et al. 2010; Peng et al. 2011; Xu et al. 2011), which is associated with their hemoglobin concentration (Beall et al. 2010; Yi et al. 2010). Network analysis indicated that the other candidate hypoxiaresponse genes we identified would all be regulated by HIF signaling pathway (Supplemental Fig. 6), suggesting an essential role of EPAS1 in the adaptation of high-altitude dogs.

The region with the second largest $F_{\mathrm{ST}}$ value $\left[Z\left(F_{\mathrm{ST}}\right)=9.75\right]$ (Fig. 2A) among the three altitude levels is overlapped with methionine sulfoxide reductase B3 (MSRB3). Both the high- and middlealtitude breeds show high differentiation to the low-altitude ones in this region $\left[Z\left(F_{\mathrm{ST}}\right)=9.77\right.$ and 6.75 , respectively] (Supplemental Fig. 5), implying that the selective sweep occurred on the migration of dogs from low to middle altitude (Supplemental Table 7). MSRB3 has been identified as a gene associated with ear types in dogs (Boyko et al. 2010; Vaysse et al. 2011), but its association with altitude has not been reported previously. The beta hemoglobin $(H B B)$ cluster region is also among the top three outliers $\left[Z\left(F_{\mathrm{ST}}\right)=8.51\right]($ Fig. $2 \mathrm{~A})$ and has a large differentiation between the high- and low-altitude breeds $\left[Z\left(F_{\mathrm{ST}}\right)=8.84\right]$ (Supplemental Fig. 5), indicating a selective sweep on the dogs' settlement from low to high altitude (Supplemental Table 7). The increases in oxygen affinity of hemoglobin have been found in diverse animal species to mediate the adaptive response to high altitude hypoxia (Storz and Moriyama 2008).

In addition to the increase of population differentiation, selective sweeps could result in the decrease of genetic diversity and increase of LD around the selective target (Nielsen 2005). Therefore, we compared $\pi$ and iHH of the six breeds around the EPAS1 (Fig. 2B) and $H B B$ loci (Fig. 2C), respectively. The $\pi$ value of TM is decreased in the EPAS1 region and is the lowest among the six breeds. The $\mathrm{iHH}$ value of TM is increased in the EPAS1 region and is the highest among the six breeds (Fig. 2B). Both of the results supported that a selective sweep occurred in the EPAS1 region in TM. The DQ breed also shows an increased $\mathrm{iHH}$ value in the EPAS1 region as TM. However, the $\pi$ value is increased in DQ, which may be caused by genetic admixture (Fig. 2B). The diversity of $\mathrm{LJ}$ in the EPAS1 region is the highest among the six breeds, suggesting extensive admixture and weak directional selection in the middle altitude dogs (Fig. 2B). When we inspected the beta hemoglobin cluster region, we observed a decrease of $\pi$ value and increase of iHH value in both TM and DQ (Fig. 2C), confirming the $F_{\mathrm{ST}}$ screening that a selective sweep occurred in the loci of high-altitude dogs.

\section{Mutations in the EPASI gene and physiological association}

We examined the mutations in the EPAS1 gene in detail and found four nonsynonymous mutations in our sample, which cause amino acid variants Gly305-Ser (G305S), Asp494-Glu (D494E), Val500-Met (V500M), and Pro750-Ser (P750S) in the translated protein (based on Ensembl annotation) (Fig. 3A; Supplemental Table 8; Flicek et al. 2014). The four mutant alleles are in complete $\mathrm{LD}$ (pairwise $r^{2}=1$ ) in all breeds, which are part of an 8-kb LD block (Supplemental Fig. 7). Haplotype tree (Fig. 3B) and network (Supplemental Fig. 7) of the LD block show that the haplotypes comprising the four mutant alleles all belong to the high-altitude clade, which have a long evolutionary distance from the haplotypes of
A

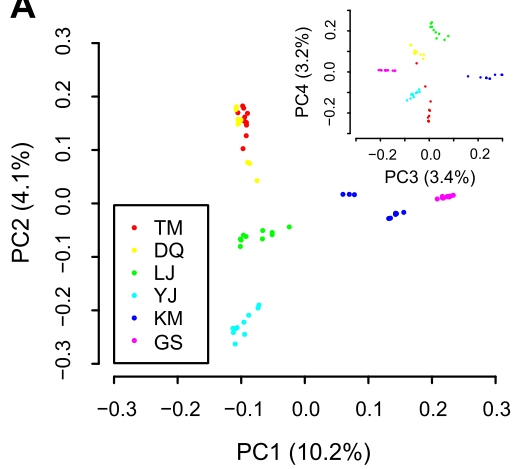

B

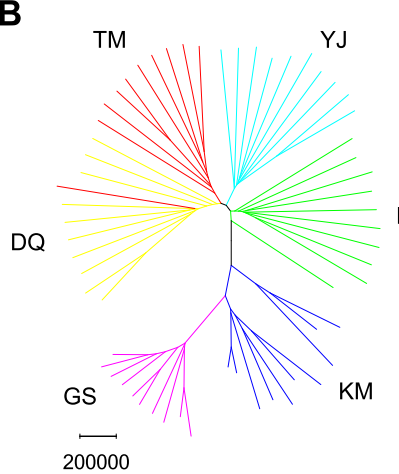

C

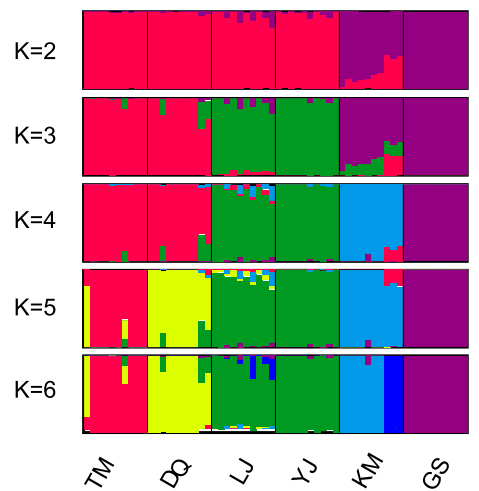

Figure 1. Genetic relationships and population structure of the 60 dogs based on all autosomal SNPs. (A) Principal component plot. The first (PC1) and second component (PC2) are shown in the main figure, while the third (PC3) and fourth component (PC4) are shown in the inset. The percentages indicate the proportion of variance explained by each component. (B) Unrooted neighbor-joining tree. The evolutionary distance is measured by the number of net nucleotide substitutions between individuals. (C) Population structures with the number of ancestral clusters $K$ from 2 to 6 . Each color represents one ancestral cluster and each vertical bar represents one dog. The length of colored segments represents corresponding ancestry attributions.

\section{Genome Research}

www.genome.org 

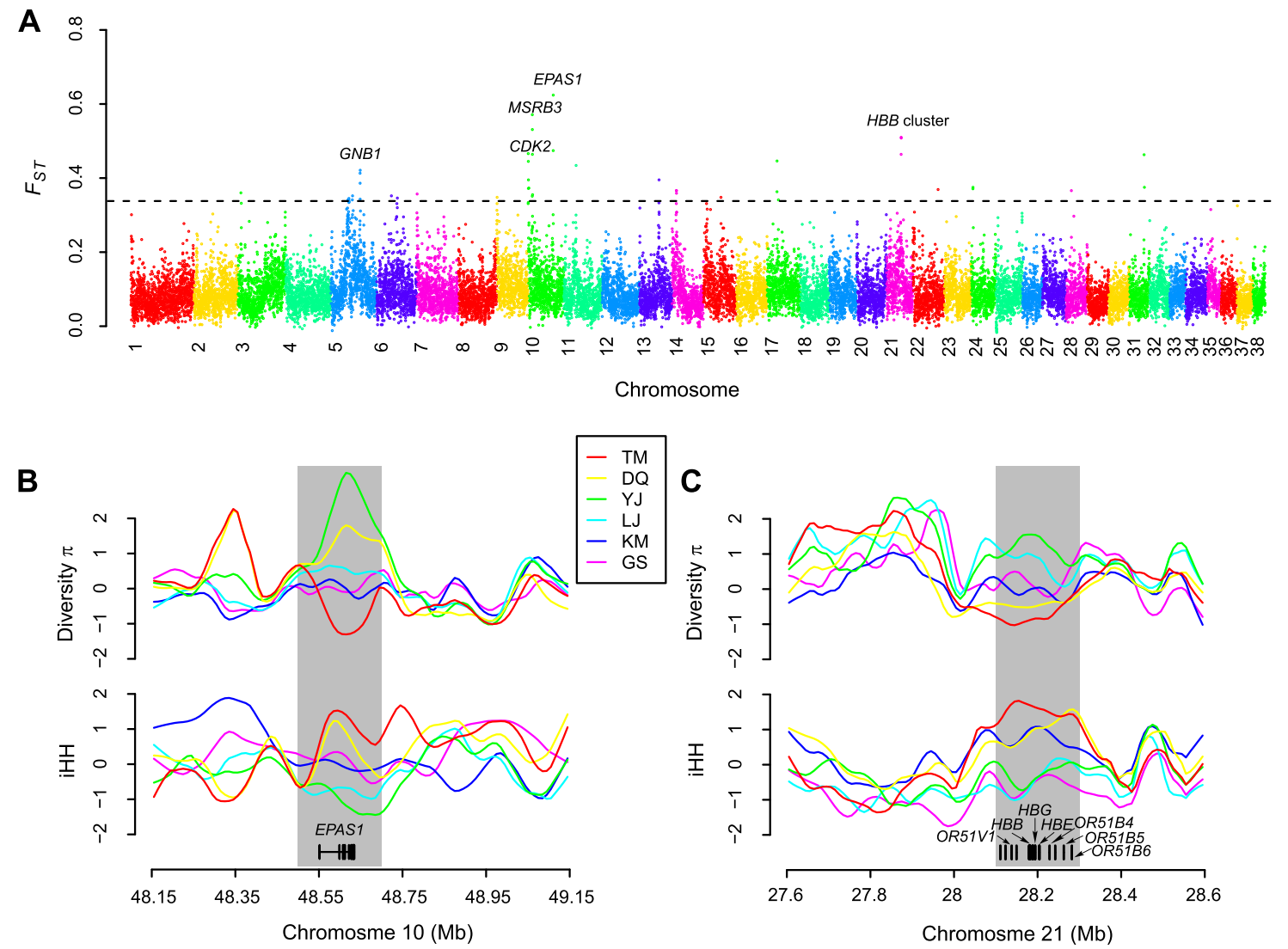

Figure 2. Selective sweep analysis of the breeds from different altitude levels. (A) Manhattan plot of $F_{\mathrm{ST}}$ among the high- (TM, DQ), middle- (LI), and low- $(\mathrm{Y}], \mathrm{KM}, \mathrm{GS})$ altitude breeds. The $F_{\mathrm{ST}}$ was calculated for each $100-\mathrm{kb}$ autosomal window. The dashed line denotes a threshold of $Z\left(F_{\mathrm{ST}}\right)=5$. $(B)$ Diversity $\pi$ and $\mathrm{iHH}$ around the EPAS1 loci. The region with $Z\left(F_{\mathrm{ST}}\right)>5$ is shaded. $\pi$ and iHH were calculated for each 10-kb window and $Z$-transformed on the genome scale. The values were smoothed by lowess regression. (C) $\pi$ and $\mathrm{iHH}$ around the beta hemoglobin loci.

the low-altitude clade. Also, the phylogenetic tree of the six breeds reconstructed from the LD block show separated clades between high and middle/low altitude (Supplemental Fig. 8), which is quite distinct from the genome-wide tree (ancient-modern separation). Interestingly, the frequency of the four mutant alleles is increased with the elevated altitude. It keeps rare in low-altitude breeds (KM 0\%, GS 0\%, YJ 5\%), moderate in middle-altitude breeds (LJ 30\%) but reaches high frequency in high-altitude breeds (DQ 80\%, TM 100\%) (Fig. 3C). To confirm this observation based on highthroughput sequencing, we performed genotyping of the four alleles on additional samples with Sanger sequencing and the result was similar: KM 0\% $(n=11)$, GS 0\% $(n=10)$, YJ 0\% $(n=20)$, LJ 20\% $(n=$ $32)$, DQ $73 \%(n=30)$, TM $94 \%(n=35)$ (Fig. 3C). The high divergence of the haplotypes and alleles among the breeds indicates that the nonsynonymous mutations may be responsible for the selective sweep at EPAS1.

Among the four variants, only one (G305S) occurred in a welldefined protein domain (PAS domain, Fig. 3A). This domain facilitates heterodimerization with the aryl hydrocarbon receptor nuclear translocator (ARNT), resulting in a functional transcriptional complex (Patel and Simon 2008). Taking the crystal structure of PAS domain in human EPAS1 as template, our homology modeling suggested that the mutation G305S occurred in a beta sheet, which may affect the thermodynamic stability of the domain (Supplemental Fig. 9). To further evaluate the functional impact of the variants, we aligned the mutant EPAS1 protein with its ortholog proteins in diverse vertebrates (Fig. 3A). The comparison reveals that G305S is also a quite conserved amino acid mutation, which is invariant among all the other animals we examined. In contrast, the remaining three variants could all be found in other species. Prediction of functional effects of the variants supports that only G305S is deleterious, while the others are tolerated (Supplemental Table 8). All of these results imply that G305S is the most likely causal mutation for the EPAS1 sweep in high-altitude dogs.

To explore the physiology of the dogs' adaptation to high-altitude hypoxia, we measured the hematologic and hemorheologic parameters for indigenous dogs living in a range from 500 to 3000 m (Supplemental Table 9). There are evident tendencies of the blood-related traits with the increase of altitude. Especially, the adult hemoglobin concentrations among middle- and high-altitude dogs reach $170 \mathrm{~g} / \mathrm{L}$ as compared with $130 \mathrm{~g} / \mathrm{L}$ among low-altitude dogs, which is a clear advantage in the rise of arterial oxygen content. However, with the increase of hemoglobin concentration, the blood viscosity and vascular resistance are also increased (Supplemental Table 9), which brings about potential risks for cardiovascular systems (Somer and Meiselman 1993; Allen and Patterson 1995). As the association between SNPs at EPAS1 and blood-related traits has been well established in humans (van Patot and Gassmann 2011), we conducted association testing for the variant G305S in DQ (Supplemental Table 10), the high-altitude breed where enough homozygotes $(n=40)$ and heterozygotes $(n=29)$ could be collected. Although no evident relationship with hemoglobin concentration 
A

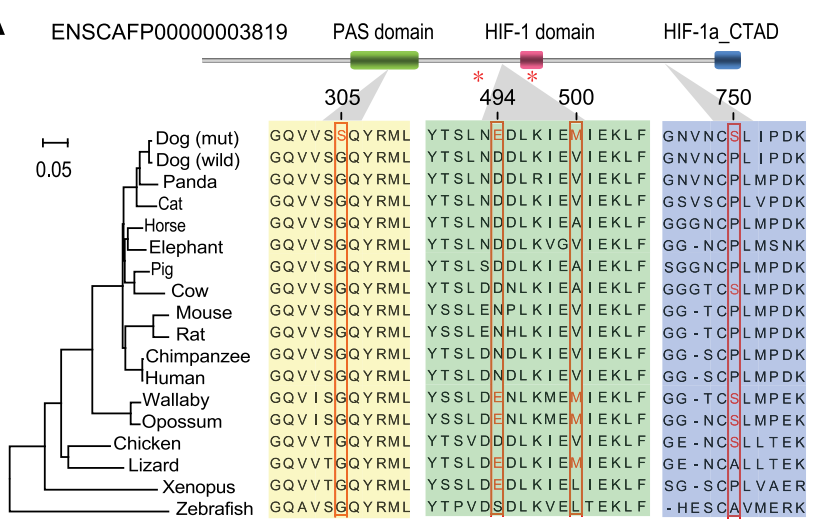

B

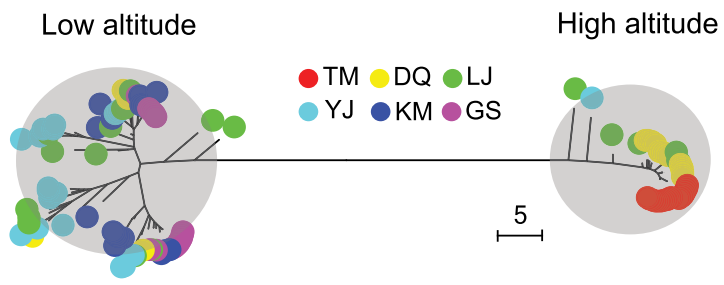

C

$100 \% 80 \quad 60 \quad 40 \quad 20 \quad 0$
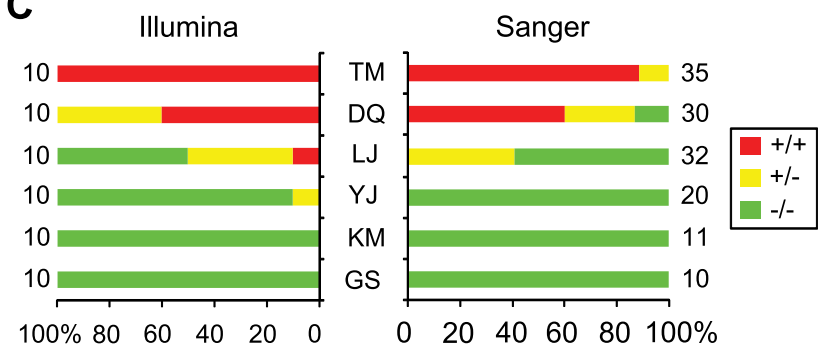

D

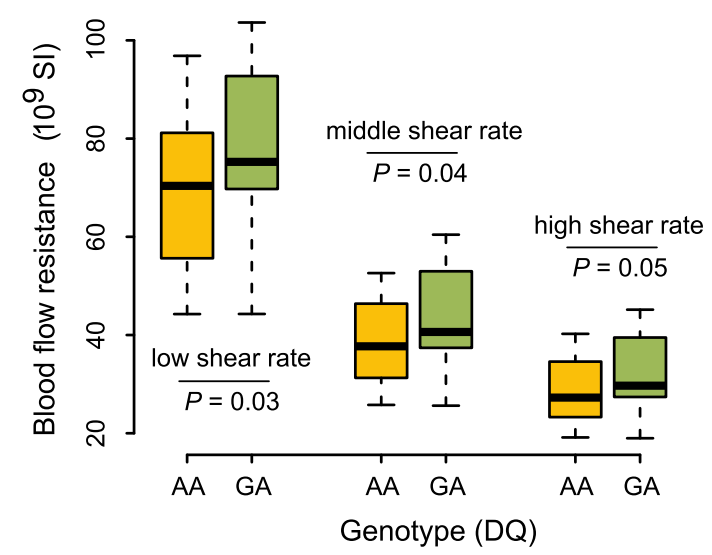

Figure 3. EPAS1 mutations in the coding region. (A) Structural and evolutionary analysis of the four amino acid variants. The protein coordinate is based on Ensembl ID ENSCAFP00000003819. The coordinate of NCBI RefSeq XP_531807.2 is slightly different but the variants remain the same. The upper panel shows the Pfam domains of the protein. The orthologous protein sequences from 17 vertebrates are aligned with the mutant residues shown in red. The NJ tree derived from the multiple alignment is shown in the left panel. The red stars indicate two hydroxylation sites (Pro405 and Pro531), which are essential for oxygen sensing (Patel and Simon 2008). (PAS) Per-Arnt-Sim; (HIF) hypoxia-inducible factor; (CTAD) C-terminal transactivation domain. (B) NJ tree of the 8-kb haplotypes comprising the four nonsynonymous mutations. The evolutionary distance is defined as the number of different nucleotides between haplotypes. The haplotypes from high- and low-altitude breeds were grouped into two distinct clades. (C) Percentages of homozygotes and heterozygotes in the six breeds. The mutant and reference alleles are represented by " + " and " - ", respectively. Besides the 60 dogs sequenced with the Illumina technology, additional samples were genotyped with Sanger sequencing. The sample size is shown beside the bars. (D) Association between EPAS1 genotypes (mutant allele, A; reference allele, $\mathrm{G}$ ) and blood flow resistance in DQ. The blood flow resistance was measured at different shear rates. In the boxplot, the line within the box defines the median; the ends of the boxes define the 25th and 75th percentiles; and the error bars define the 10th and 90 th percentiles. The ANOVA F-test was performed, taking the age as covariant.

was found, we discovered a statistically significant association between the genotypes and vascular resistance (Fig. 3D). The homozygotes with two mutant alleles show decreased vascular resistance as compared with the heterozygotes. The results suggest that the G305S variant may play important roles in the improvement of blood fluidity, which confers advantages for oxygen delivery and cardiovascular functions.

\section{Discussion}

In this study, we performed whole-genome sequencing of 60 dogs including five breeds from continuous altitudes along the Ancient Tea Horse Road in the Tibetan Plateau as well as one breed of GS. We catalogued millions of SNPs of each breed for evolutionary and genetic researches. Specifically, this is the first study to characterize the genetic polymorphisms of TM, which are known as "oriental deiform dogs" in Tibet and one of the most ancient and ferocious dogs in the world (Li and Zhang 2012). Our population structure analyses revealed that TM have a close but different genetic background with other indigenous dogs living at high altitude (Fig. 1), though their origin and relationship with plateau wolves need further studies. We also found the existence of admixture among TM and indigenous dogs (Fig. 1), which raised the demands for genetic testing in the identification and breeding of TM.
We conducted selective sweep mapping for the breeds from different altitudes and identified several candidate regions with a high extent of differentiation on the genome scale (Fig. 2A). Two of the genes contained in the top regions, EPAS1 and $H B B$, were previously reported as targets of natural selection for hypoxia adaptation in humans and other animals (Storz and Moriyama 2008; van Patot and Gassmann 2011). The two loci also displayed reduced genetic diversity and/or increased LD in high-altitude dogs especially TM (Fig. 2B,C). These results suggest that, driven by the same hypoxic environment, natural selection could act on a similar group of genes in the adaptive process of different species. Interestingly, the parallel evolution on the molecular level may extensively exist between humans and dogs in metabolic and neurological processes, probably due to their similar environments in the recent past (Wang et al. 2013).

We discovered and validated four novel nonsynonymous mutations at EPAS1 in high-altitude dogs, of which G305S occurred in a well-defined domain and quite conserved site (Fig. 3A). The mutant alleles and corresponding haplotypes have a large divergence between low- and high-altitude dogs (Fig. 3B,C), implying that the amino acid variations may be responsible for the signal of selective sweep at the EPAS1 locus.

The transcription factor encoded by EPAS1 plays a key role in transcriptional response to hypoxia in various physiological and

\section{Genome Research} www.genome.org 
pathologic conditions, including up-regulating the expression of erythropoietin (EPO) (Patel and Simon 2008). Especially, mutations at EPAS1 in humans are strongly associated with hematologic phenotypes (van Patot and Gassmann 2011). On one hand, clinical case studies found that several gain-of-function mutations occurred in close vicinity to the primary hydroxylation site (Pro531) at EPAS1 cause erythrocytosis (Percy et al. 2008a,b). On the other hand, the EPAS1 polymorphisms unique to the native Tibetan people were found to be associated with their lower hemoglobin concentrations (Beall et al. 2010; Yi et al. 2010), suggesting a loss-of-function role of EPAS1 in high-altitude adaptation. As erythrocytosis is a common symptom of chronic mountain sickness which will lead to high blood viscosity and cardiovascular disorders, the decrease in hemoglobin level may provide a protective mechanism for Tibetan people (Beall et al. 2010; van Patot and Gassmann 2011). In fact, comparing with Andeans and other highland migrants with shorter settlement history, Tibetans do not show markedly elevated hemoglobin concentration (Beall 2007), probably due to their unique EPAS1 genotypes (van Patot and Gassmann 2011). Instead, Tibetans display a higher blood flow for oxygen delivery (Erzurum et al. 2007), which could compensate the effect of lower hemoglobin concentration.

Nonetheless, it is worth noting that all EPAS1 variations detected previously in the Tibetan population are at introns (Beall et al. 2010; Yi et al. 2010), inhibiting further study of its molecular mechanism in the adaptive process. Fortunately, the key amino acid mutation we identified at EPAS1 in dogs, G305S, is predicted to be damaging, which is also likely to cause the loss of function of EPAS1. Moreover, we demonstrated that the variation is associated with lower blood flow resistance in high-altitude dogs (Fig. 3D). Therefore, we propose that similar mechanisms may be adopted by both humans and dogs in adaptation to high-altitude hypoxia. Although the physiological responses need further comparison between humans and dogs, these novel variations of EPAS1 could shed new insights into the study of the gene function.

\section{Methods}

\section{Sample preparation and sequencing}

For each dog, the genomic DNA was extracted from $200 \mu \mathrm{L}$ peripheral venous blood with the QIAamp DNA Blood Mini kit (Qiagen). The quality and integrity of DNA was controlled by A260/280 ratio and agarose gel electrophoresis. For sequencing library preparation, the genomic DNA was sheared to fragments of 300-500 bp, which were then end-repaired, " $\mathrm{A}$ "-tailed, and ligated to Illumina sequencing adapters. The ligated products with sizes of $400-500$ bp were selected on $2 \%$ agarose gels and then amplified by LM-PCR. The libraries were sequenced on Illumina HiSeq 2000 with $2 \times 100$ bp paired-end mode, which was controlled by Illumina HiSeq Control Software.

\section{Reads processing and variant calling}

The raw reads were processed by two rounds of quality control (QC) (Supplemental Table 1). Firstly, low quality reads marked by Illumina sequencers in the FASTQ files (Casava v1.8) were filtered. Secondly, for each read, the low quality end with base quality scores $<20$ were trimmed. The read was removed if its length was $<35$ after trimming. Only paired reads were preserved during QC. Reads after QC were mapped to the dog reference genome assembly canFam3 (http://hgdownload.soe.ucsc.edu/goldenPath/canFam3/ bigZips/canFam3.fa.gz) using BWA-MEM (v0.7.4) (Li and Durbin 2009) with default parameters on a per-individual basis. We re- moved duplicated reads and calculated mapping statistics including the coverage of depth from the .bam files by SAMtools (v0.1.19) (Li et al. 2009; Supplemental Table 2).

The SNPs and small indels were called using the SAMtools pipeline (Li 2011) on a per-breed (10 individuals) basis (Supplemental Table 3). Firstly, .mpileup files were generated by SAMtools mpileup with the parameters "-u -C50 -DS -q20". Secondly, .vcf files containing raw variants were generated by bctools view with the parameters "-evcgN". Finally, the raw variants were filtered by vcfutils.pl varFilter with the minimum depth "-d 20" and maximum depth "-D 300". The cutoffs were set according to the sequencing depth distribution per individual (Supplemental Fig. 1). Other parameters for filtering were default, including $P$-value of HardyWeinberg equilibrium (0.0001). Given the position of a variant, the genotype with the maximum posterior probability of each individual was given in the .vcf file. The variants after filtering were annotated by ANNOVAR (v2013-06-21) (Wang et al. 2010) according to Ensembl (Flicek et al. 2014) gene annotation (http://hgdownload. soe.ucsc.edu/goldenPath/canFam3/database/ensGene.txt.gz) (Supplemental Tables 4, 5).

\section{Population genetics analysis}

The .vcf files for the six breeds were merged by vcf-merge in VCFtools (v0.1.11) (Danecek et al. 2011). When a genotype was missing in one breed, it was set to the reference allele with the option "-R 0/0". Only biallelic SNPs in autosomes were preserved for subsequent population structure and polymorphism analysis. The merged .vcf file was converted to tab-delimited text file by vcfto-tab in VCFtools, and to PLINK format files (.ped and .map) by VCFtools and PLINK (v1.07) (Purcell et al. 2007) when appropriate. The PCA was performed by GCTA (v1.13) (Fig. 1A; Yang et al. 2011), which first generated the genetic relationship matrix (GRM) with the "-make-grm" option and then estimated the first four principal components with the "-pca 4" option. The pairwise genetic distances between individuals were measured by the number of net nucleotide substitutions (formula 12.67 in Nei and Kumar 2000), which were calculated for each SNP sites and then summed up. Based on the distance matrix, the NJ tree was constructed and displayed by MEGA (v5.2) (Fig. 1B; Tamura et al. 2011). The population structure was inferred by frappe (v1.1) (Tang et al. 2005) with a maximum likelihood method (Fig. 1C). The number of ancestral clusters $K$ ranged from 2 to 6 , with 10,000 iterations each run. The graphs of the population structure were displayed by distruct (v1.1) (Rosenberg 2004) on a per-individual basis.

Standard population genetic statistics, including Watterson's $\theta$, pairwise nucleotide diversity $\pi$ (Supplemental Fig. 2), and Tajima's $D$ (Supplemental Fig. 3) were calculated for each breed by the Bio: :PopGen: :Statistics package in BioPerl (v1.6.1) (Stajich et al. 2002). The coefficient of nucleotide differentiation $F_{\mathrm{ST}}$ among the breeds was calculated by the Bio:PopGen: PopStats package in BioPerl (Supplemental Table 6). The statistics were estimated over a sliding window with a fixed size along the genome. The Manhattan plot of genome-wide $F_{\mathrm{ST}}$ values was generated using the mhtplot function in the R package gap (Fig. 2A; Supplemental Fig. 5). The level of LD measured by the correlation coefficient $\left(r^{2}\right)$ between unphased markers (Supplemental Fig. 4A) was calculated by Haploview (v4.2) (Barrett et al. 2005) with the option "-dprime -maxdistance 50 -minMAF 0.1 -hwcutoff 0.05 ". We also phased the genotypes of all individuals into haplotypes by fastPHASE (v1.4.0) (Scheet and Stephens 2006) with the parameters "-T10 -K8 - $u$ ". The iHH (Voight et al. 2006) for each breed was calculated by XP-EHH (Pickrell et al. 2009) based on the phased data (Supplemental Fig. 4B). The $\mathrm{iHH}$ values were log-transformed and averaged over a sliding window with fixed size along the genome. 


\section{EPAS1 mutation analysis}

The protein domains of EPAS1 were predicted by the Pfam web service (Fig. 3A; Punta et al. 2012). The ortholog proteins of EPAS1 in 17 representative vertebrates were retrieved from Ensembl (Flicek et al. 2014) (release 72) and only one-to-one orthologs were included (Fig. 3A). The multiple sequence alignment was performed by ClustalX (v2.1) (Larkin et al. 2007) and displayed by Jalview (v2.8) (Waterhouse et al. 2009). The LD block containing the nonsynonymous mutations was detected and visualized by Haploview (v4.2) (Supplemental Fig. 7A; Barrett et al. 2005). The haplotype NJ tree was constructed by MEGA (v5.2) (Tamura et al. 2011) with the default parameters (Fig. 3B). The haplotype network was constructed and plotted by Network (v4.611, Fluxus Engineering) (Supplemental Fig. 7B). It was calculated with the median-joining method (Bandelt et al. 1999) $(\varepsilon=0)$, followed by reduction with the MP option (Polzin and Daneshmand 2003). The sequence of dog EPAS1 was searched against the PDB database (Rose et al. 2013) using BLAST-P (Altschul et al. 1997) to identify suitable templates for homology modeling (Supplemental Fig. 9). I-TASSER (v2.1) (Roy et al. 2010) was used for structure modeling by threading the sequence onto the selected templates. The three-dimensional model structures were visualized with PyMOL (v1.6, Schrödinger, LLC). Structure-based energy calculation was performed by the Eris server (Yin et al. 2007) using the flexible backbone method upon single amino acid substitutions. The functional consequences of amino acid mutations were predicted by SIFT (Kumar et al. 2009), which was invoked through Ensembl's (Flicek et al. 2014) Variant Effect Predictor (Supplemental Table 8).

\section{Physiological measurement and association test}

The foreleg venous blood was collected to measure the physiological parameters in situ. The hematologic and hemorheologic parameters were measured by BC-2800Vet Auto Hematology Analyzer (Mindray Co., Ltd.) and ZL1000 Auto Blood Rheology Analyzer (Zonci Co., Ltd.), respectively (Supplemental Tables 9, 10). The nonsynonymous mutations at EPAS1 were genotyped by traditional Sanger sequence technology. The association testing was performed with each physiological parameter as the response variable, genotype as the predictive variable, and age $(<1$ yr vs. $>1$ yr) as the covariate. We also modeled the gender as a covariate but no difference was found in this factor. The $F$ test in the analysis of variance (ANOVA) was used to evaluate the significance of genotypes (Fig. 3D).

\section{Data access}

All sequencing data from this study have been submitted to the NCBI Sequence Read Archive (SRA; http://www.ncbi.nlm.nih.gov/ sra) under accession number SRP035294.

\section{Acknowledgments}

We thank Professor Li Yang from CAS-MPG Partner Institute for Computational Biology, SIBS, CAS, and Dr. Zhongkai Gu from the Institute of Biomedical Sciences, Fudan University for their help in providing sequencing instruments. We also thank the anonymous reviewers for their constructive comments. We are indebted to many people whose names are not included in the author list, but who contributed to this project. This work was supported by the State Key Basic Research Program (973) (2011CB910204, 2010CB529206, 2011CBA00801), Research Program of CAS (KSCX2-EW-R-04, KSCX2-YW-R-190, XXH12503-0202-09), National Natural Science Foundation of China (31260544,
U1036604, 31070752, 31301032), Chinese Ministry for Science and Technology Grant (2008BAI64B01), Chinese High-Tech R\&D Program (863) (2013AA102503, 2009AA02Z304, 2012AA020404), China Postdoctoral Science Foundation (2013M531226), Shanghai Postdoctoral Scientific Program (13R21417300), and the SA-SIBS Scholarship Program.

\section{References}

Akey JM, Ruhe AL, Akey DT, Wong AK, Connelly CF, Madeoy J, Nicholas TJ, Neff MW. 2010. Tracking footprints of artificial selection in the dog genome. Proc Natl Acad Sci 107: 1160-1165.

Alkorta-Aranburu G, Beall CM, Witonsky DB, Gebremedhin A, Pritchard JK, Di Rienzo A. 2012. The genetic architecture of adaptations to high altitude in Ethiopia. PLoS Genet 8: e1003110.

Allen MT, Patterson SM. 1995. Hemoconcentration and stress: a review of physiological mechanisms and relevance for cardiovascular disease risk. Biol Psychol 41: 1-27.

Altschul SF, Madden TL, Schaffer AA, Zhang J, Zhang Z, Miller W, Lipman DJ. 1997. Gapped BLAST and PSI-BLAST: a new generation of protein database search programs. Nucleic Acids Res 25: 3389-3402.

Axelsson E, Ratnakumar A, Arendt ML, Maqbool K, Webster MT, Perloski M, Liberg O, Arnemo JM, Hedhammar A, Lindblad-Toh K. 2013. The genomic signature of dog domestication reveals adaptation to a starchrich diet. Nature 495: 360-364.

Bandelt HJ, Forster P, Rohl A. 1999. Median-joining networks for inferring intraspecific phylogenies. Mol Biol Evol 16: 37-48.

Barrett JC, Fry B, Maller J, Daly MJ. 2005. Haploview: analysis and visualization of LD and haplotype maps. Bioinformatics 21: 263-265.

Beall CM. 2007. Two routes to functional adaptation: Tibetan and Andean high-altitude natives. Proc Natl Acad Sci (Suppl 1) 104: 8655-8660.

Beall CM, Laskowski D, Strohl KP, Soria R, Villena M, Vargas E, Alarcon AM, Gonzales C, Erzurum SC. 2001. Pulmonary nitric oxide in mountain dwellers. Nature 414: 411-412.

Beall CM, Decker MJ, Brittenham GM, Kushner I, Gebremedhin A, Strohl KP. 2002. An Ethiopian pattern of human adaptation to high-altitude hypoxia. Proc Natl Acad Sci 99: 17215-17218.

Beall CM, Cavalleri GL, Deng L, Elston RC, Gao Y, Knight J, Li C, Li JC, Liang Y, McCormack M, et al. 2010. Natural selection on EPAS1 (HIF2 $\alpha$ ) associated with low hemoglobin concentration in Tibetan highlanders. Proc Natl Acad Sci 107: 11459-11464.

Bigham AW, Mao X, Mei R, Brutsaert T, Wilson MJ, Julian CG, Parra EJ, Akey JM, Moore LG, Shriver MD. 2009. Identifying positive selection candidate loci for high-altitude adaptation in Andean populations. Hum Genomics 4: 79-90.

Bigham A, Bauchet M, Pinto D, Mao XY, Akey JM, Mei R, Scherer SW, Julian CG, Wilson MJ, Herraez DL, et al. 2010. Identifying signatures of natural selection in Tibetan and Andean populations using dense genome scan data. PLoS Genet 6: e1001116.

Boyko AR, Quignon P, Li L, Schoenebeck JJ, Degenhardt JD, Lohmueller KE, Zhao K, Brisbin A, Parker HG, vonHoldt BM, et al. 2010. A simple genetic architecture underlies morphological variation in dogs. PLoS Biol 8: e1000451.

Cho YS, Hu L, Hou H, Lee H, Xu J, Kwon S, Oh S, Kim HM, Jho S, Kim S, et al. 2013. The tiger genome and comparative analysis with lion and snow leopard genomes. Nat Commun 4: 2433.

Danecek P, Auton A, Abecasis G, Albers CA, Banks E, DePristo MA, Handsaker RE, Lunter G, Marth GT, Sherry ST, et al. 2011. The variant call format and VCFtools. Bioinformatics 27: 2156-2158.

Erzurum SC, Ghosh S, Janocha AJ, Xu W, Bauer S, Bryan NS, Tejero J, Hemann C, Hille R, Stuehr DJ, et al. 2007. Higher blood flow and circulating NO products offset high-altitude hypoxia among Tibetans. Proc Natl Acad Sci 104: 17593-17598.

Flicek P, Amode MR, Barrell D, Beal K, Billis K, Brent S, Carvalho-Silva D, Clapham P, Coates G, Fitzgerald S, et al. 2014. Ensembl 2014. Nucleic Acids Res 42: D749-D755.

Ge RL, Cai QL, Shen YY, San A, Ma L, Zhang Y, Yi X, Chen Y, Yang LF, Huang Y, et al. 2013. Draft genome sequence of the Tibetan antelope. Nat Commun 4: 1858.

Jessen TH, Weber RE, Fermi G, Tame J, Braunitzer G. 1991. Adaptation of bird hemoglobins to high altitudes: demonstration of molecular mechanism by protein engineering. Proc Natl Acad Sci 88: 6519-6522.

Kumar P, Henikoff S, Ng PC. 2009. Predicting the effects of coding nonsynonymous variants on protein function using the SIFT algorithm. Nat Protoc 4: 1073-1081.

Larkin MA, Blackshields G, Brown NP, Chenna R, McGettigan PA, McWilliam H, Valentin F, Wallace IM, Wilm A, Lopez R, et al. 2007. Clustal W and Clustal X version 2.0. Bioinformatics 23: 2947-2948. 
Li H. 2011. A statistical framework for SNP calling, mutation discovery, association mapping and population genetical parameter estimation from sequencing data. Bioinformatics 27: 2987-2993.

Li H, Durbin R. 2009. Fast and accurate short read alignment with BurrowsWheeler transform. Bioinformatics 25: 1754-1760.

Li Y, Zhang YP. 2012. High genetic diversity of Tibetan Mastiffs revealed by mtDNA sequences. Chin Sci Bull 57: 1483-1487.

Li H, Handsaker B, Wysoker A, Fennell T, Ruan J, Homer N, Marth G, Abecasis G, Durbin R. 2009. The Sequence Alignment/Map format and SAMtools. Bioinformatics 25: 2078-2079.

Li M, Tian S, Jin L, Zhou G, Li Y, Zhang Y, Wang T, Yeung CK, Chen L, Ma J, et al. 2013. Genomic analyses identify distinct patterns of selection in domesticated pigs and Tibetan wild boars. Nat Genet 45: 1431-1438.

Liang Y, Hua Z, Liang X, Xu Q, Lu G. 2001. The crystal structure of barheaded goose hemoglobin in deoxy form: the allosteric mechanism of a hemoglobin species with high oxygen affinity. J Mol Biol 313: 123137.

Nei M, Kumar S. 2000. Molecular evolution and phylogenetics. Oxford University Press, New York.

Nielsen R. 2005. Molecular signatures of natural selection. Annu Rev Genet 39: $197-218$

Patel SA, Simon MC. 2008. Biology of hypoxia-inducible factor- $2 \alpha$ in development and disease. Cell Death Differ 15: 628-634.

Peng Y, Yang Z, Zhang H, Cui C, Qi X, Luo X, Tao X, Wu T, Ouzhuluobu, Basang, et al. 2011. Genetic variations in Tibetan populations and highaltitude adaptation at the Himalayas. Mol Biol Evol 28: 1075-1081.

Percy MJ, Beer PA, Campbell G, Dekker AW, Green AR, Oscier D, Rainey MG, van Wijk R, Wood M, Lappin TR, et al. 2008a. Novel exon 12 mutations in the HIF2A gene associated with erythrocytosis. Blood 111: 54005402.

Percy MJ, Furlow PW, Lucas GS, Li X, Lappin TR, McMullin MF, Lee FS. 2008 b. A gain-of-function mutation in the HIF2A gene in familial erythrocytosis. N Engl J Med 358: 162-168.

Piccinini M, Kleinschmidt T, Jurgens KD, Braunitzer G. 1990. Primary structure and oxygen-binding properties of the hemoglobin from guanaco (Lama guanacoe, Tylopoda). Biol Chem Hoppe Seyler 371: 641648.

Pickrell JK, Coop G, Novembre J, Kudaravalli S, Li JZ, Absher D, Srinivasan BS, Barsh GS, Myers RM, Feldman MW, et al. 2009. Signals of recent positive selection in a worldwide sample of human populations. Genome Res 19: 826-837.

Polzin T, Daneshmand SV. 2003. On Steiner trees and minimum spanning trees in hypergraphs. Oper Res Lett 31: 12-20.

Punta M, Coggill PC, Eberhardt RY, Mistry J, Tate J, Boursnell C, Pang N, Forslund K, Ceric G, Clements J, et al. 2012. The Pfam protein families database. Nucleic Acids Res 40: D290-D301.

Purcell S, Neale B, Todd-Brown K, Thomas L, Ferreira MA, Bender D, Maller J, Sklar P, de Bakker PI, Daly MJ, et al. 2007. PLINK: a tool set for wholegenome association and population-based linkage analyses. Am J Hum Genet 81: 559-575.

Qiu Q, Zhang GJ, Ma T, Qian WB, Wang JY, Ye ZQ, Cao CC, Hu QJ, Kim J, Larkin DM, et al. 2012. The yak genome and adaptation to life at high altitude. Nat Genet 44: 946.

Rose PW, Bi C, Bluhm WF, Christie CH, Dimitropoulos D, Dutta S, Green RK, Goodsell DS, Prlic A, Quesada M, et al. 2013. The RCSB Protein Data Bank: new resources for research and education. Nucleic Acids Res $\mathbf{4 1}$ D475-D482.

Rosenberg NA. 2004. DISTRUCT: a program for the graphical display of population structure. Mol Ecol Notes 4: 137-138.

Roy A, Kucukural A, Zhang Y. 2010. I-TASSER: a unified platform for automated protein structure and function prediction. Nat Protoc 5: 725 738.

Scheet P, Stephens M. 2006. A fast and flexible statistical model for largescale population genotype data: applications to inferring missing genotypes and haplotypic phase. Am J Hum Genet 78: 629-644.

Scheinfeldt LB, Soi S, Thompson S, Ranciaro A, Woldemeskel D, Beggs W, Lambert C, Jarvis JP, Abate D, Belay G, et al. 2012. Genetic adaptation to high altitude in the Ethiopian highlands. Genome Biol 13: R1.
Simonson TS, Yang Y, Huff CD, Yun H, Qin G, Witherspoon DJ, Bai Z, Lorenzo FR, Xing J, Jorde LB, et al. 2010. Genetic evidence for highaltitude adaptation in Tibet. Science 329: 72-75.

Somer T, Meiselman HJ. 1993. Disorders of blood viscosity. Ann Med 25: $31-$ 39.

Stajich JE, Block D, Boulez K, Brenner SE, Chervitz SA, Dagdigian C, Fuellen G Gilbert JG, Korf I, Lapp H, et al. 2002. The Bioperl toolkit: Perl modules for the life sciences. Genome Res 12: 1611-1618.

Storz JF, Moriyama H. 2008. Mechanisms of hemoglobin adaptation to high altitude hypoxia. High Alt Med Biol 9: 148-157.

Storz JF, Sabatino SJ, Hoffmann FG, Gering EJ, Moriyama H, Ferrand N, Monteiro B, Nachman MW. 2007. The molecular basis of high-altitude adaptation in deer mice. PLoS Genet 3: e45.

Storz JF, Runck AM, Sabatino SJ, Kelly JK, Ferrand N, Moriyama H, Weber RE, Fago A. 2009. Evolutionary and functional insights into the mechanism underlying high-altitude adaptation of deer mouse hemoglobin. Proc Natl Acad Sci 106: 14450-14455.

Tamura K, Peterson D, Peterson N, Stecher G, Nei M, Kumar S. 2011. MEGA5: molecular evolutionary genetics analysis using maximum likelihood, evolutionary distance, and maximum parsimony methods. Mol Biol Evol 28: 2731-2739.

Tang H, Peng J, Wang P, Risch NJ. 2005. Estimation of individual admixture: analytical and study design considerations. Genet Epidemiol 28: 289-301.

van Patot MCT, Gassmann M. 2011. Hypoxia: adapting to high altitude by mutating EPAS-1, the gene encoding HIF-2 $\alpha$. High Alt Med Biol 12: 157167.

Vaysse A, Ratnakumar A, Derrien T, Axelsson E, Rosengren Pielberg G, Sigurdsson S, Fall T, Seppala EH, Hansen MS, Lawley CT, et al. 2011. Identification of genomic regions associated with phenotypic variation between dog breeds using selection mapping. PLoS Genet 7: e1002316.

Voight BF, Kudaravalli S, Wen X, Pritchard JK. 2006. A map of recent positive selection in the human genome. PLoS Biol 4: e72.

Vonholdt BM, Pollinger JP, Lohmueller KE, Han E, Parker HG, Quignon P, Degenhardt JD, Boyko AR, Earl DA, Auton A, et al. 2010. Genome-wide SNP and haplotype analyses reveal a rich history underlying dog domestication. Nature 464: 898-902.

Wang K, Li M, Hakonarson H. 2010. ANNOVAR: functional annotation of genetic variants from high-throughput sequencing data. Nucleic Acids Res 38: e164.

Wang GD, Zhai WW, Yang HC, Fan RX, Cao X, Zhong L, Wang L, Liu F, Wu H, Cheng LG, et al. 2013. The genomics of selection in dogs and the parallel evolution between dogs and humans. Nat Commun 4: 1860 .

Waterhouse AM, Procter JB, Martin DM, Clamp M, Barton GJ. 2009. Jalview Version 2-a multiple sequence alignment editor and analysis workbench. Bioinformatics 25: 1189-1191.

Weber RE, Lalthantluanga R, Braunitzer G. 1988. Functional characterization of fetal and adult yak hemoglobins: an oxygen binding cascade and its molecular basis. Arch Biochem Biophys 263: 199-203.

Xu S, Li S, Yang Y, Tan J, Lou H, Jin W, Yang L, Pan X, Wang J, Shen Y, et al. 2011. A genome-wide search for signals of high-altitude adaptation in Tibetans. Mol Biol Evol 28: 1003-1011.

Yang J, Lee SH, Goddard ME, Visscher PM. 2011. GCTA: a tool for genomewide complex trait analysis. Am J Hum Genet 88: 76-82.

Yi X, Liang Y, Huerta-Sanchez E, Jin X, Cuo ZX, Pool JE, Xu X, Jiang H, Vinckenbosch N, Korneliussen TS, et al. 2010. Sequencing of 50 human exomes reveals adaptation to high altitude. Science 329: 75-78.

Yin S, Ding F, Dokholyan NV. 2007. Eris: an automated estimator of protein stability. Nat Methods 4: 466-467.

Zhang J, Hua Z, Tame JR, Lu G, Zhang R, Gu X. 1996. The crystal structure of a high oxygen affinity species of haemoglobin (bar-headed goose haemoglobin in the oxy form). J Mol Biol 255: 484-493.

Zhao M, Kong QP, Wang HW, Peng MS, Xie XD, Wang WZ, Jiayang, Duan JG, Cai MC, Zhao SN, et al. 2009. Mitochondrial genome evidence reveals successful Late Paleolithic settlement on the Tibetan Plateau. Proc Natl Acad Sci 106: 21230-21235.

Received December 30, 2013; accepted in revised form April 8, 2014. 


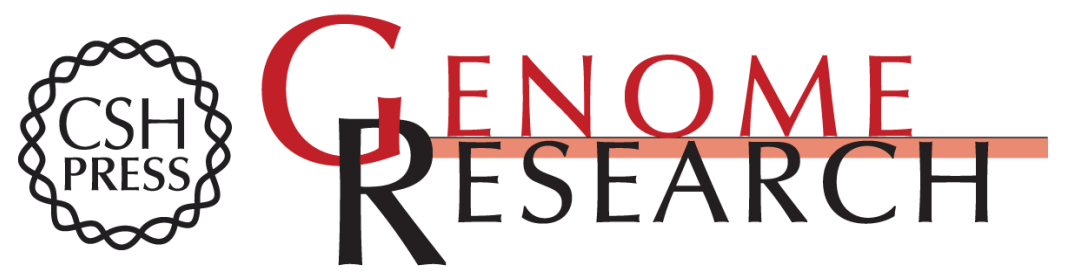

\section{Whole-genome sequencing of six dog breeds from continuous altitudes reveals adaptation to high-altitude hypoxia}

Xiao Gou, Zhen Wang, Ning Li, et al.

Genome Res. 2014 24: 1308-1315 originally published online April 10, 2014

Access the most recent version at doi:10.1101/gr.171876.113

\section{Supplemental} Material

References

Creative

Commons

License

Email Alerting

Service
http://genome.cshlp.org/content/suppl/2014/06/10/gr.171876.113.DC1

This article cites 66 articles, 13 of which can be accessed free at: http://genome.cshlp.org/content/24/8/1308.full.html\#ref-list-1

This article is distributed exclusively by Cold Spring Harbor Laboratory Press for the first six months after the full-issue publication date (see

$\mathrm{http}: / / g$ enome.cshlp.org/site/misc/terms.xhtml). After six months, it is available under a Creative Commons License (Attribution-NonCommercial 4.0 International), as described at http://creativecommons.org/licenses/by-nc/4.0/.

Receive free email alerts when new articles cite this article - sign up in the box at the top right corner of the article or click here.

\section{Affordable, Accurate Sequencing.}

